Original research article

Food Quality and Functionality Section

\title{
Approaches for Improvement in Digestive Survival of Probiotics, a Comparative Study
}

\author{
Agnieszka Dudkiewicz, ${ }^{1,2}$, Laura Masmejean ${ }^{1,3}$, Clément Arnaud ${ }^{1,3}$, Bukola Adenike \\ Onarinde ${ }^{1}$, Ramana Sundara ${ }^{4}$, Amir Hossein Pour-Taghi Anvarian ${ }^{1}$, Nick Tucker ${ }^{*}$ \\ ${ }^{1}$ University of Lincoln, National Centre for Food Manufacturing, Park Road, PE12 7 PT, Holbeach, UK \\ 2Jan Dlugosz University in Czestochowa, Faculty of Science and Technology, 13/15 Aleja Armii Krajowej, \\ 42-200, Czestochowa, Poland \\ ${ }^{3}$ Polytech Montpellier, University of Montpellier, Place Eugène Bataillon, CC419, 34095 Montpellier, France \\ ${ }^{4}$ University of Lincoln, Lincoln Institute for Agri-food Technology, Brayford Pool, LN6 7TS, Lincoln, UK \\ ${ }^{5}$ University of Lincoln, School of Engineering, Brayford Pool, LN6 7TS, Lincoln, UK
}

Key words: probiotics, prebiotics, encapsulation, food matrices, in vitro digestion, survival in digestive tract

The aim of this study was to compare approaches commonly recommended in the literature for the improvement of the survival of probiotics in the human digestive tract. The survival of two probiotics, Lactobacillus casei and Bifidobacterium lactis, in the presence or absence of prebiotics, maize starch, fermented milk and upon encapsulation in calcium alginate-chitosan was evaluated. While $B$. lactis was resistant to stomach juice, but sensitive to duodenal juice, $L$. casei showed an exactly opposite behaviour. In contrast to other published studies, here the overall digestive survivability of probiotics was not improved significantly by prebiotics, maize starch or encapsulation. However, a significant improvement of the overall survivability of $B$. lactis (but not $L$. casei) during in vitro digestion was noted in milk and fermented milk, possibly due to reduction of the activity of bile against this probiotic. Summarising, no one method could be universally recommended for the improvement of probiotic survivability. Nevertheless, this research indicated that certain probiotic characteristics, such as susceptibility to bile or acid or ability to utilise matrix components as an energy source, could be used in further research to select the most effective approaches to deliver viable cells into lower parts of the digestive tract.

\section{INTRODUCTION}

Delivering live probiotics to the lower part of the human digestive tract, when administered orally, presents a challenge. Low stomach $\mathrm{pH}$ and action of bile salt hamper their survival [Bezkorovainy, 2001].

According to multiple studies, the survivability of probiotics in the digestive tract can be improved by application of a food matrix or addition of a prebiotic, or encapsulation [Burgain et al., 2011; Govender et al., 2014; Kingwatee et al., 2014; Sanchez et al., 2014]. As yet, these three methods were not confirmed to ensure effective survival of multiple strains of probiotics. Previous studies have examined the behaviour of only one or very few probiotics without consideration for differences in their survival characteristics [e.g. Burgain et al., 2011; Haghshenas et al., 2015; Kingwatee et al., 2014; Lo Curto et al., 2011; Sanchez et al., 2014; Sumeri et al., 2010]. Additionally, these studies varied in the application of in vitro digestion models which could be dynamic or static and applied different $\mathrm{pH}$, digestion time, and chemical concentrations at each stage of digestion, making comparison difficult. Therefore, based on such data it is not possible to distinguish

\footnotetext{
* Corresponding Author: E-mail: ntucker@lincoln.ac.uk (Dr. N. Tucker) juice but unstable in duodenum juice (unlike B. lactis W52).

the method for oral route delivery of probiotics, with the best protective effect. This is important since many of the commercial probiotic formulations combine multiple strains.

The few existing studies comparing different approaches for the improvement of probiotic survivability focus on combined effects, e.g., encapsulation with addition of elements of food matrix or prebiotic [Fredua-Agyeman \& Gaisford, 2015; Shori, 2017]. Such studies also do not allow a conclusion as to whether there is a general method to improve the digestive survivability of probiotics.

Therefore, in this study we compared three methods previously shown to improve probiotic survival and two probiotic strains featuring different sensitivity to digestive juices.

The three compared methods were:

- Food matrix inclusion (fresh and fermented milk, maize starch),

- Different prebiotic concentrations (inulin and FOS),

- Electrospray encapsulation in calcium alginate and chitosan.

The chosen probiotic strains were Lactobacillus casei W56 and Bifdobacterium lactis W52, which according to the manufacturer should feature different sensitivities to gastro-intestinal conditions. L. casei W56 was sensitive to stomach 
Thus, it was possible to test the protective effect of the applied methods to probiotics in stomach and duodenal conditions. Ideally, the method which would be generally recommended for the improvement of the probiotic survival should be effective in both types of digestive juices. Testing whether such an approach was available from those recommended by the literature was the subject of this study.

\section{MATERIALS AND METHODS}

\section{Bacterial strains, materials, and reagents}

Two probiotic strains used in the study, B. lactis W52 and L. casei W56, in a powdered form, were obtained from Winclove Probiotics (Amsterdam, Netherlands). Based on inhouse experiments conducted by Winclove Probiotics, it was expected that $B$. lactis W52 would survive the in vitro digestion better compared to L. casei W56. Freeze-dried organisms were kept at $4^{\circ} \mathrm{C}$ prior to use. Winclove Probiotics supplied commercial prebiotics - Fructo-oligosaccharide (FOS P1) (hereafter FOS), and inulin containing a small quantity of FOS (Inulin and FOS P7) (hereafter inulin), and a probiotic supplement carrier material (Maize Starch) that were used as received. Full fat milk (composition per $100 \mathrm{~mL}$ : fat $3.6 \mathrm{~g}$, sugar $4.7 \mathrm{~g}$, and protein $3.4 \mathrm{~g}$; pH 6.8) was purchased from a local supermarket. Sodium chloride, sodium bicarbonate, potassium chloride, calcium chloride, porcine pepsin, porcine pancreatin, and porcine bile used for the digestive assay were obtained from Fisher (Loughborough, UK). Sodium alginate, chitosan, and calcium carbonate used in probiotic encapsulation were purchased from Avonchem limited (Macclesfield Cheshire, UK), Acros Organics (Morris Plains, USA) and Fisher (Loughborough, UK), respectively.

\section{Preparation of samples challenged to in vitro digestion}

Portions of approximately $0.1 \mathrm{~g}$ of probiotic powders were combined with prebiotics (at four levels of concentration: $0.1,0.5,1$, and $5 \%, w / w)$ or maize starch $(5 \%, w / w)$ by mixing in sterile tap water. Probiotics were added aseptically. The count of cells in the initial sample was above $8 \mathrm{log}$ $\mathrm{cfu} / \mathrm{mL}$ (count of viable cells in each sample is presented in the Results and Discussion section).

To allow resuscitation of probiotics, samples were kept for $15 \mathrm{~min}$ at room temperature prior to analysis and commencement of the simulation of digestion. The highest concentration of prebiotic was chosen to reflect the dose that was previously shown to have a beneficial effect to the human health [Kellow et al., 2014]. Fermented milk was prepared by the incubation of sterile milk $(150 \mathrm{~mL})$ with the addition of $\sim 0.1 \mathrm{~g}$ of probiotic powder with either L. casei W56 or B. lactis W52 for $18 \mathrm{~h}$ at $40^{\circ} \mathrm{C}$. The $\mathrm{pH}$ of milk following fermentation was 4.3 for L. casei W56 and 4.7 for B. lactis W52. Control samples contained only sterile tap water and probiotics.

For probiotic encapsulation, we have chosen calcium alginate additionally coated with chitosan. Calcium alginate is a frequently used encapsulation material, since it is food-grade and enables release of the probiotics in intestines [Segale et al., 2016]. However, it tends to create porous capsules, which may mean that the probiotics would not be protected from the contact with digestive juices [Burgain et al.,
2011]. To minimise this effect and enhance the survival, calcium alginate capsules were coated with chitosan following the example of Shori [2017].

Consideration was also given to the method of capsule preparation. One of the novel methods being increasingly applied in research studies is electrospraying, where voltage is used to extrude a polymer solution through a capillary, resulting in a formation of small droplets [Coghetto et al., 2016]. This method is suitable for use with probiotics as compared to some other methods, such as e.g. spray-drying, it does not require high temperatures.

For encapsulation, probiotics were mixed into the $22.5 \mathrm{~g} / \mathrm{kg}$ sodium alginate solution. They were either added to the sodium alginate as a supplied freeze-dried powder $(\sim 0.1 \mathrm{~g}$ of powder to $5 \mathrm{~mL}$ of alginate; powder) or upon previous resuscitation (broth). The resuscitation was carried out by subsequently:

1. Inoculating $150 \mathrm{~mL}$ de Man Rogosa Sharpe (MRS) broth with $\sim 0.1 \mathrm{~g}$ of probiotic powder and incubating for $24 \mathrm{~h}$ at $37^{\circ} \mathrm{C}$ (B. lactis was grown in MRS broth supplemented with $0.5 \mathrm{~g} / \mathrm{L}$ L-cysteine),

2. Harvesting cells from $40 \mathrm{~mL}$ of the broth by centrifugation at $1500 \times g$ for $15 \mathrm{~min}$ at $25^{\circ} \mathrm{C}$,

3. Washing and centrifuging the pellet twice with the saline solution using same settings as in 2., and

4. Re-suspending the pellet in $3 \mathrm{~mL}$ of the saline solution and adding to $20 \mathrm{~mL}$ of the alginate solution.

Suspensions of probiotics in sodium alginate were then electrosprayed. The electrospraying process has been explained in the previous publication [Zaeim et al., 2017]. The equipment used for electrospraying was provided by Electrospinz Ltd (Blenheim, New Zealand). The set up was composed of a polymer header tank, a hollow needle with a $0.06 \mathrm{~mm}$ internal diameter, and a dish collector grounded through a crocodile clip. The solution was electrosprayed at $8 \mathrm{kV}$ and the distance between the needle and the collector was $8 \mathrm{~cm}$. The polymer/ probiotic solution was placed in the header tank and flowed under gravity to the needle. Droplets were electrostatically attracted into a dish collector which contained $500 \mathrm{mM}$ calcium carbonate solution at $\mathrm{pH}$ 5.2. About $0.5 \mathrm{~g}$ of calcium alginate droplets encapsulating probiotics were obtained during a single $2 \mathrm{~h}$ run. Capsules were then filtered through a filter paper (Whatman no 4, Fisher, Loughborough, UK), rinsed with sterile water, and further coated with chitosan.

For coating, $20 \mathrm{mg}$ of chitosan was dissolved in $2 \mathrm{~mL}$ of $100 \mathrm{~mL} / \mathrm{L}$ glacial acetic acid and the $\mathrm{pH}$ was raised to $\sim 6.0$ by adding $0.5 \mathrm{M} \mathrm{NaOH}$. Alginate micro beads were immersed in the chitosan solution and stirred at $60 \mathrm{rpm}$ for 40 min using an orbital shaker (LSE, Corning, New York, US). This procedure was adapted from a method by Sohail et al. [2011].

The coated capsules were then filtered, washed twice with sterile water, and placed in a fresh portion of sterile water. The capsule suspension was stored for up to 2 days at $4^{\circ} \mathrm{C}$ prior to digestion assay.

Capsules were prepared in triplicate and characterised by means of the optical microscope (MOTIC B1 Advanced Series with Motic Images Plus version 3 software for im- 
age analysis). An example image of these capsules is shown in Figure 1. The prepared capsules contained probiotics at a level of $>6 \log \mathrm{cfu} / \mathrm{g}$.

\section{In vitro digestion model}

To investigate the survivability of probiotics in the human digestive tract, a model designed based on several studies was applied. The composition and proportions of the digestive juices were adapted from studies of Marteau et al. [1997] and Timmerman et al. [2007] and were given in Table 1. The chosen transit times were $30 \mathrm{~min}$ in the stomach, $1 \mathrm{~h}$ in the duodenum and $2 \mathrm{~h}$ in the ileum. The $\mathrm{pH}$ during digestion was 2 for stomach and 6.5 for duodenum stage. Ileal juice was simulated by the addition of $11.5 \mathrm{mM}$ of $\mathrm{CaCl}_{2}$ to duodenum juice containing the sample in order to deactivate bile salts. Anaerobiosis during the digestion was created by overlying digestive liquids with $5 \mathrm{~mL}$ of mineral oil. Anaerobiosis seemed to be applied only in recent artificial digestion studies on the survival of probiotics, nevertheless it is a realistic condition present in the digestive tract. In our preliminary assessment, we saw a better survival of the microorganisms, especially L. casei W56 in the stomach juice, when anaerobiosis was applied. Stomach and duodenal juice were prepared and warmed up to $37^{\circ} \mathrm{C}$ prior to experimentation. Artificial digestion was carried out at $37^{\circ} \mathrm{C}$ with a constant agitation on an orbital shaker (LSE, Corning, New York, US) at 60 RPM.

Liquid samples were added in volumes of $5 \mathrm{~mL}$, whilst capsules in quantities of $\sim 0.5 \mathrm{~g}$ suspended in $5 \mathrm{~mL}$ of sterile deionised water to digestive juices. The $\mathrm{pH}$ of digestive juices was measured and re-adjusted upon the addition of the samples. licate.

In vitro digestion of each sample was carried out in trip-

\section{Enumeration of probiotics}

Enumeration of lactic acid bacteria in the samples was carried out using the ISO 15214:1998 method. Briefly, samples were serially diluted in buffered peptone water (BPW, Oxoid, Basingstoke, England, CM0509). The diluted samples were then transferred in volume of $1 \mathrm{~mL}$ to empty Petri dishes and mixed with $\sim 15 \mathrm{~mL}$ of de Man, Rogosa, Sharpe agar (MRS, Oxoid, Basingstoke, England, CM0361). Growth of B. lactis W52 was encouraged by the addition of $0.5 \mathrm{~g} / \mathrm{L}$ of $\mathrm{L}$-cysteine into MRS agar. Plates were incubated at $37^{\circ} \mathrm{C}$ for $72 \mathrm{~h}$ in $150 \mathrm{~mL} / \mathrm{L} \mathrm{CO}_{2},<10 \mathrm{~mL} / \mathrm{L} \mathrm{O}_{2}$ and $\mathrm{N}_{2}$ atmosphere created with the MULTIVAC T200 tray sealer (Multivac, Wolfertschwenden, Germany). The limit of detection for the method was $1 \mathrm{cfu} / \mathrm{mL}$ of digestive juice. Results below the limit of detection were included in statistical analysis as $0.5 \mathrm{cfu} / \mathrm{mL}$.

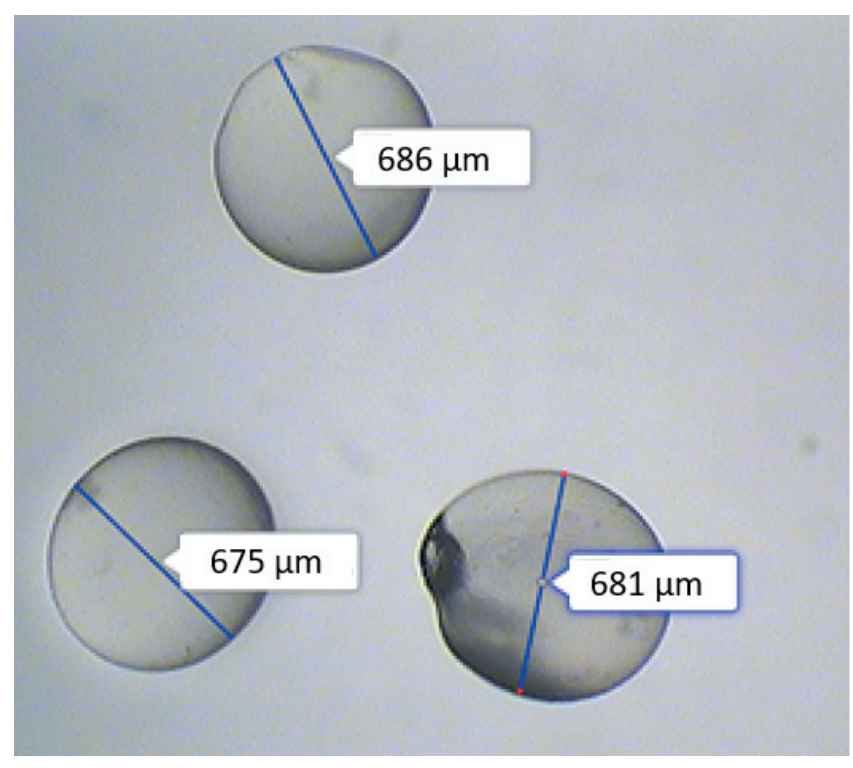

FIGURE 1. Light microscopy image of capsules obtained in the process of electrospraying.

Calcium alginate-chitosan capsules were visually intact through the entire digestion process. Hence, prior to enumeration, cells were released from the capsules. This was carried out by stirring encapsulated probiotics in $10 \mathrm{~mL}$ of $0.1 \mathrm{M}$ phosphate buffer at $\mathrm{pH} 7$ at room temperature for $30 \mathrm{~min}$ on a magnetic stirrer. The release of the probiotics from capsules has been confirmed by preliminary trials, where the count of viable cells was determined at different time points during mixing.

\section{Statistical analysis}

Statistical tests were performed using IBM SPSS Statistics 22. All assumed a significance level of 0.05. Particular tests are mentioned in the Results and Discussion section next to relevant data.

All $\log$ reduction values quoted in the text and shown in the figures were corrected for the dilution factors caused by the addition of stomach and duodenum juice to the samples within the digestive assay.

\section{RESULTS AND DISCUSSION}

\section{The survival of probiotics during in vitro digestion}

Survival curves of L. casei W56 and B. lactis W52 during passage through digestive liquids in control sample, as well as in the presence of prebiotics, food matrices, and in encapsulates was presented in Figure 2. Statistical tests (repeated measures

TABLE 1. Composition of simulated stomach and duodenal juice.

\begin{tabular}{|c|c|c|c|c|c|c|c|}
\hline & $\mathrm{NaCl}$ & $\mathrm{NaHCO}_{3}$ & $\mathrm{KCl}$ & $\mathrm{CaCl}_{2}$ & $\begin{array}{c}\text { Porcine } \\
\text { pepsin }\end{array}$ & $\begin{array}{c}\text { Porcine } \\
\text { pancreatin }\end{array}$ & $\begin{array}{c}\text { Porcine } \\
\text { bile }\end{array}$ \\
\hline & \multicolumn{7}{|c|}{$(\mathrm{g} / \mathrm{L})$} \\
\hline Stomach & 6.2 & 1.2 & 2.2 & 0.22 & 3.2 & - & - \\
\hline Duodenum & 5 & - & 0.6 & 0.25 & - & 9 & 14 \\
\hline
\end{tabular}


A

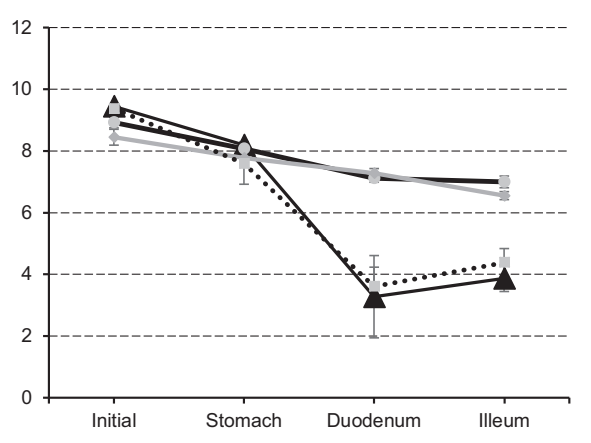

B

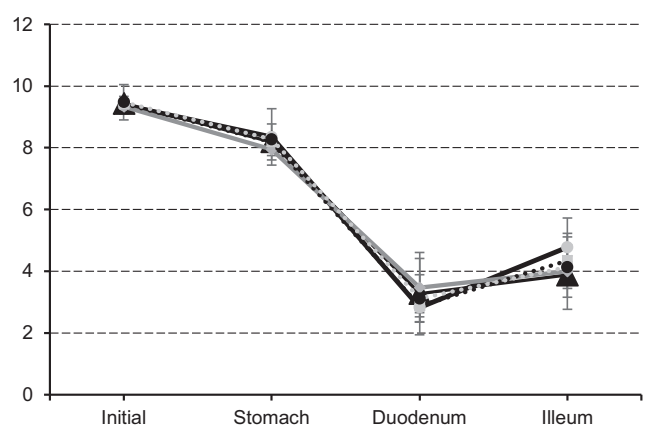

C

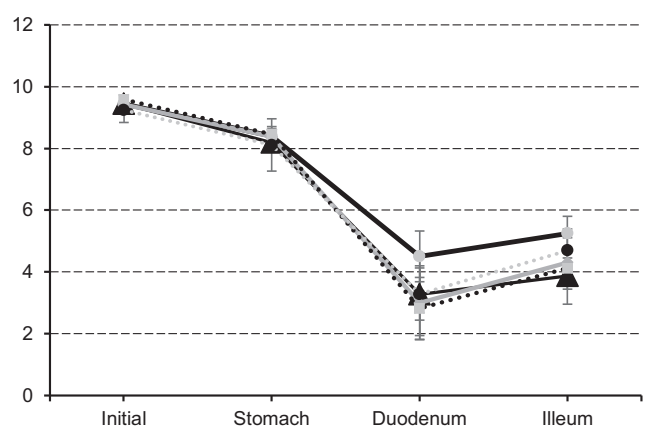

$\rightarrow$ Control

$-0.10 \%$

$\longrightarrow 0.50 \%$

....... $1 \%$

- $.5 \%$

-Control

$-0.10 \%$

$\longrightarrow 0.50 \%$

....... $1 \%$

- $\cdots 5 \%$

D

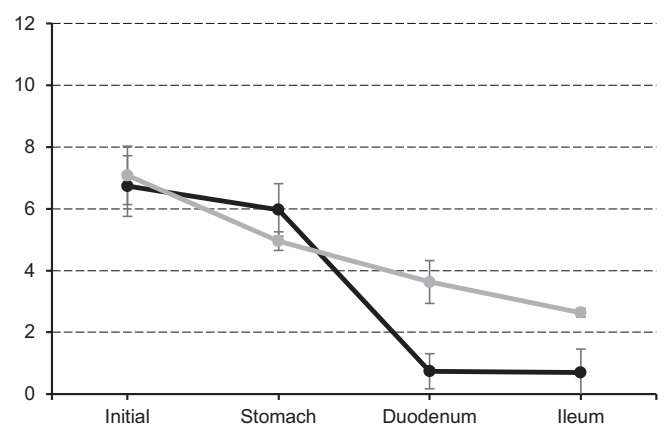

E

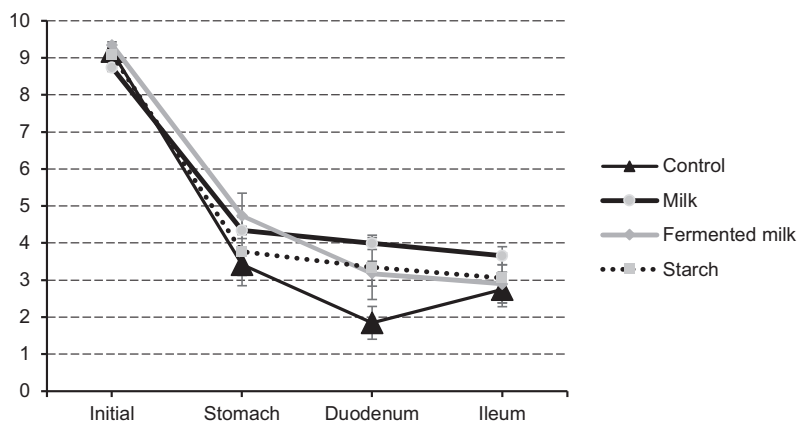

$\mathrm{F}$

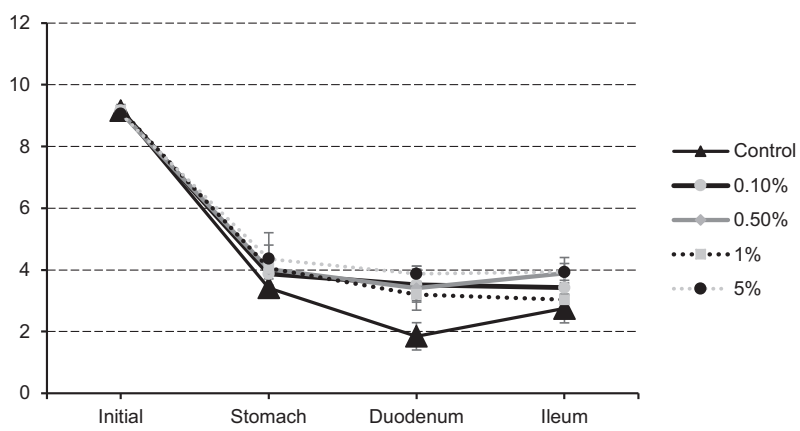

G

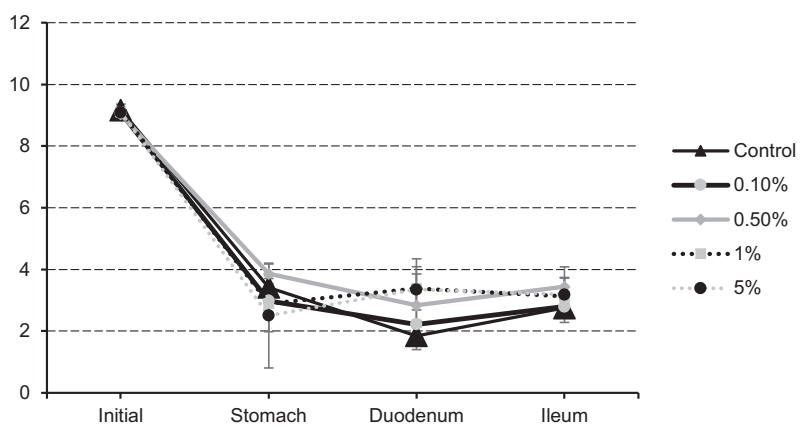

$\mathrm{H}$

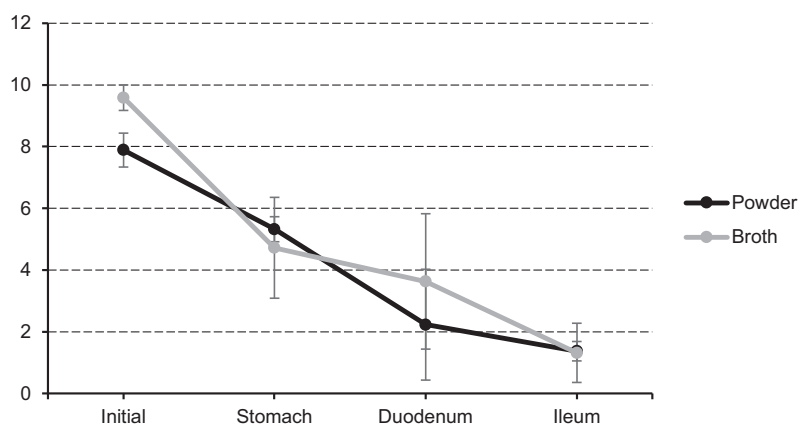

FIGURE 2. Survival during in vitro digestion of B. lactis W52 (graphs A, B, C and D) and L. casei W56 (graphs E, F, G and H) in presence of (A and E) food matrices, (B and F) inulin, (C and G) FOS, and (D and H) in capsules. Errors bars correspond to standard deviation. Counts are expressed per $\mathrm{mL}$ of initial solution. Dilutions factors from the addition of juices during the experiment were not compensated for. 
ANOVA with Bonferroni post-hoc, results were not presented), indicated that stage in the digestion process had a significant effect on the log reduction of probiotic population. The digestion stage where the largest log reduction occurred was stomach for L. casei W56 (mean log reduction \pm standard deviation, $4.4 \pm 1.1 \log \mathrm{cfu})$ and duodenum for B. lactis W52 (3.9 $\pm 1.9 \log$ $\mathrm{cfu})$. At the same time, $L$. casei W56 seemed relatively resistant to duodenum juice $(0.6 \pm 1.4 \mathrm{log} \mathrm{cfu})$ and B. lactis W52 to stomach juice $(0.5 \pm 0.5 \log \mathrm{cfu})$. The ileum juice offered the gentlest conditions for both probiotics $(0.2 \pm 1.1$ and $-0.7 \pm 1.2 \mathrm{log}$ cfu for $L$. casei W56 and B. lactis W52 respectively, negative log reduction indicated growth).

For L. casei W56, the survival curves representing control, food matrices, and all levels of prebiotics followed a similar pattern. Encapsulated, freeze-dried L. casei W56 seemed to reduce at similar rates through all digestive stages, indicating that the approach was able to minimise the effect of stomach juice on the probiotic. However, this trend was not observed in broth-grown, encapsulated L. casei W56, where a greater decline of probiotic population in the stomach was observed. The result indicates that the form in which $L$. casei W56 was encapsulated, rather than the encapsulation, had an influence on its survivability in the stomach. In turn, $B$. lactis W52 behaved similarly to the control when probiotic was challenged to digestive assay in the presence of maize starch, prebiotics at all concentration levels as well as within capsules containing probiotic powder. The survival curves of $B$. lactis W52 in the presence of milk and fermented milk as well as upon encapsulation of the broth-grown probiotics, resembled a straight line, meaning that the decline of the probiotics was similar through all the digestion stages. Out of these three treatments, encapsulation of the broth-grown probiotics seemed to feature a steeper decline for the population of B. lactis W52 compared to when the probiotic was challenged in milk or fermented milk.

Presented survival curves are real log cfu/mL counts disregarding the dilution of the probiotics by the addition of stomach and duodenum juices. To compare the effectiveness of different approaches, total log reductions were calculated based on the concentration of probiotics in the first (initial) and after passage through the last (ileum) stage correcting for dilution factors resulting from the addition of digestive juices (see Figure 3).

Total log reductions for $L$. casei W56 ranged from 4.2 to $7.4 \mathrm{log}$ cfu (milk and capsules containing broth-grown probiotics, respectively) and for B. lactis W52 from 1.0 to $5.1 \mathrm{log}$ cfu (fermented milk and capsules containing probiotic powder, respectively). For $L$. casei W56, there was no treatment which decreased the total log reduction significantly compared to the control. However, encapsulation of broth-grown probiotics caused a significant $(\mathrm{p}<0.05)$ increase of the total log reduction compared to the control and all other treatments. On the other hand, a significantly $(\mathrm{p}<0.05)$ greater survival of B. lactis W52 was obtained in the presence of milk and fermented milk compared to the control and all the other treatments.

Overall, mean total log reductions were significantly higher for L. casei W56 compared to B. lactis W52 (paired for treatments t-test, $\mathrm{p}<0.05$ ), indicating that $B$. lactis W52 was more resistant to conditions of the digestive tract than L. casei W56.
This was in line with the suggestion given by the probiotic provider, as specified in the method section.

\section{The effect of probiotic type on the survival during in vitro digestion}

This study evaluated survivability of two different probiotic bacteria, L. casei W56 and B. lactis W52, in human digestive juices. Other works suggest that these two probiotic species might exhibit contrasting survival in human digestive tract, although it should be noted that each of these works used a different in vitro digestion design [Fávaro-Trindade \& Grosso, 2002; Kingwatee et al., 2014; Lo Curto et al., 2011].

Here, by application of a single in vitro digestion design for these two probiotic species, we had an opportunity to verify the difference in their survival. We have found that overall B. lactis W52 survived better compared to L. casei W56. Nevertheless, the magnitude of the differences in survival was affected by the type of the matrix surrounding probiotics.

\section{The effect of the presence of food matrices on the survival of probiotics during in vitro digestion}

The presence of a food matrix, such as milk, may substantially improve the survival of probiotics. Several authors noted that fat could potentially enhance probiotic survival. For example, Tompkins et al. [2011] showed that probiotics (ProtecFlor®, commercial supplement containing 4 probiotic strains) survived better in $1 \%$ fat milk and oats compared to fruit juice and spring water. Furthermore, Lo Curto et al. [2011] showed that the digestive survivability of two different probiotics: L. casei immunitas and L. acidophilus johnsonii, was improved in the presence of the whole milk matrix compared to water by 6.5 and $1 \log$ cfu, respectively. This finding indicated that different probiotics may not be equally protected by fat. In the present study, food matrices containing fat, milk, and fermented milk, improved significantly the survival of B. lactis W52 (on average by 3.6 and $3.7 \log$ cfu, respectively), but not that of $L$. casei W56 (on average by 1.3 and $0.0 \log$ cfu, respectively). Since B. lactis W52 was sensitive to duodenum juice, while L. casei W56 was comparatively resistant to it, results might point at the role of fat in the protection of probiotics from bile. Given that the task of bile is to emulsify the fat to aid its digestion, inclusion of fat into probiotic matrix could mean that the bile salts would not be free to interact with probiotic cells [Begley et al., 2005].

Based on the published literature, it was expected that fermentation of milk might add to the protective effect of the food matrix through:

1. Possible acid adaptation, especially of stomach juice sensitive L. casei W56, and

2. Propagation of the probiotic population from logarithmic growth phase into stationary phase.

Improvement of acid resistance might be expected, since the $\mathrm{pH}$ of fermented milk was lower than that of fresh milk (4.3 and 6.8, respectively). Nevertheless, in our study, the digestive survival of $L$. casei W56 was on average worse (although not significantly) in fermented milk compared to milk (total $\log$ reduction 5.5 and $4.2 \log \mathrm{cfu}$, respectively). In contrast, the development of acid adaptation was demonstrated for L. acidophilus LA-5 and L. rhamnosus GG which after ex- 
A

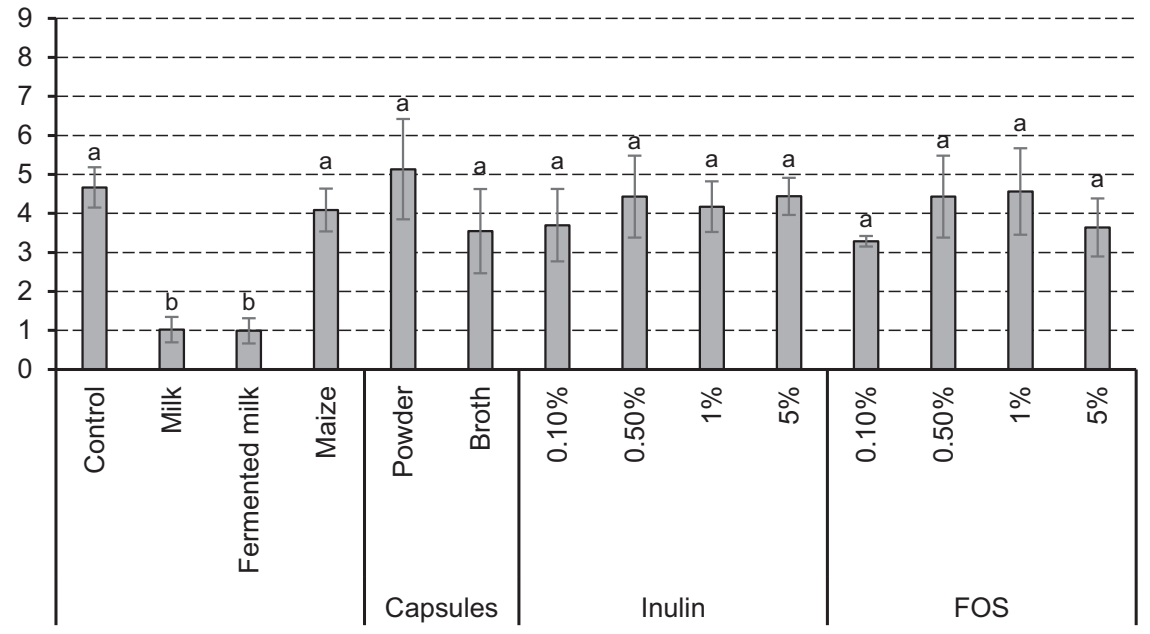

B

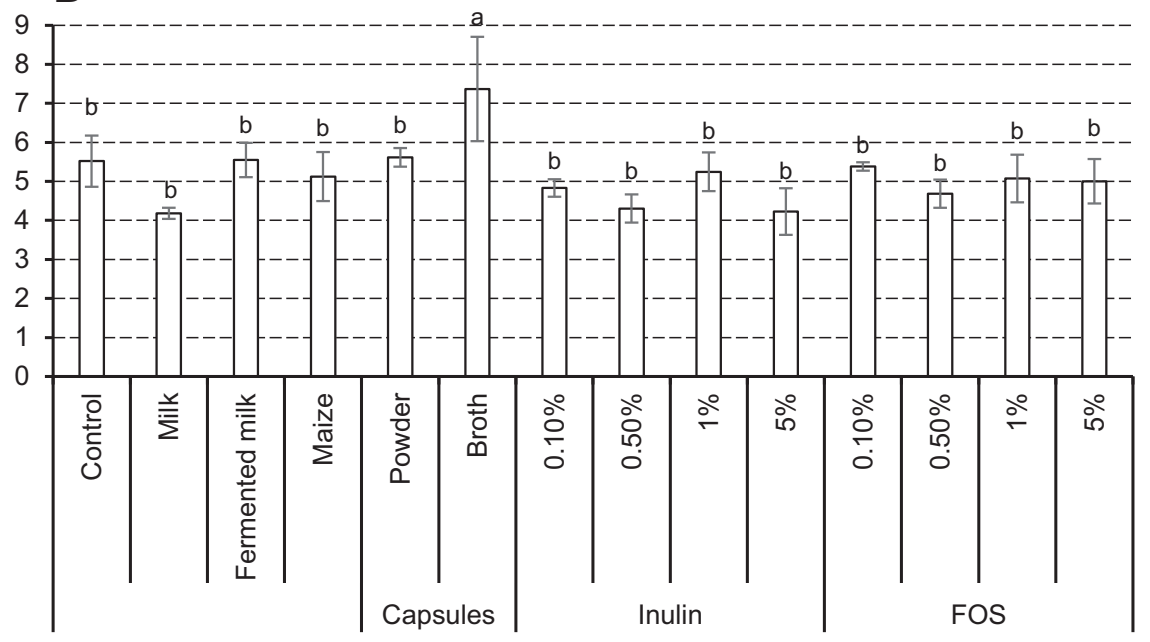

FIGURE 3. Total log reduction for (A) B. lactis W52 and (B) L. casei W56 in the in vitro assay. Columns note mean values and error bars standard deviations. Different letters above different columns in the same graph mean significant differences between different matrices and/ or control (ANOVA and Tukey post-hoc, significance level of 0.05 ).

posure to low $\mathrm{pH}$ (3.5) prior to digestion, showed a slightly better survival in the stomach acid compared to the control (by 0.31 and $0.30 \log$ cfu, respectively [Sumeri et al., 2010]). The same study did not report acid adaptation in these strains, when probiotics were adapted at $\mathrm{pH} 4.5$, which was closer to $\mathrm{pH}$ of fermented milk here, and hence supports our finding [Sumeri et al., 2010].

In fermented milk, cells should have reached the stationary growth phase. According to Lo Curto et al. [2011], probiotics challenged with digestive juices survive better when in the stationary phase compared to the logarithmic growth phase. Here, this effect was not observed for either of the two tested probiotics. Some possible reasons for different results between current and the Lo Curto et al. [2011] study are differences in used probiotic strains, digestive assay design as well as the way in which cells have been grown into the stationary phase. In the cited study, after the addition of probiotics to milk or water, samples were maintained at $4-6^{\circ} \mathrm{C}$ for 6 days, while here, the milk was fermented for $18 \mathrm{~h}$ at $40^{\circ} \mathrm{C}$ and samples were subjected to the digestive as- say, without chilling. It is not clear whether cold storage could improve the resistance of probiotics to digestive juices, nevertheless some information in support of this hypothesis can be found in the literature. It is known that the temperature of cell growth will affect the expression of genes and the physiological characteristics of microorganisms [Spano \& Massa, 2006]. Additionally, cases of the resistance to multiple stressors upon adaptation to a single stressor have been documented in the literature. For example, acid adaptation of Bifidobacterium breve resulted in a better survival during cold storage of probiotic as well as during exposure to bile and hydrogen peroxide [Park et al., 1995]. Overall, these data suggested that adaptation to cold stress could be beneficial in aiding probiotic survival during passage through the upper digestive tract.

Maize starch is used by probiotic manufacturers as a carrier material at a concentration of approx. $90 \%$ in the powdered product formulation (Winclove probiotics, private communication). In this study, digestive survival of $L$. casei W56 and B. lactis W52 in the presence of maize starch was not improved compared to the control. However, high amylose 
maize starch has been shown to enhance the survival of $\mathrm{Bi}$ fidobacterium Lafti $^{\mathrm{TM}} 8 \mathrm{~B}$ and $13 \mathrm{~B}$ during exposure to in vitro as well as in vivo (mice) digestion [Wang et al., 1999]. Significantly, both of these probiotics had an ability to utilise amylose from maize starch. The mechanism of the improvement of the digestive survival of probiotics in the presence of food source was well explained by Corcoran et al. [2005]. These authors found that metabolizable sugars have been shown to improve the tolerance of L. rhamnosus GG to acid by provision of the energy for the exclusion of protons from cells. Summarising, studies suggest that the ability of probiotics to use a present matrix as an energy source might be a factor allowing to improve their digestive survival. This was partly supported by findings in our study. The total log reduction of stomach juice-sensitive L. casei W56 was on average lowest (although not significantly) in the presence of milk compared to all other treatments (including fermented milk where the lactose concentration would be lower), suggesting that the lactose present in milk could have aided survival of this probiotic in the stomach juice.

\section{The effect of the encapsulation on the survival of probiotics during in vitro digestion}

In the present study, we have compared two encapsulation approaches - powder and broth. Most of the reviewed studies have used freshly grown and harvested cells for encapsulation (broth method). Encapsulation of the powder might however make a commercial sense, since the population of encapsulated probiotics declines during storage [Yeung et al., 2016].

Capsules obtained in this study measured between $\sim 500$ to $800 \mu \mathrm{m}$ and on average $660 \mu \mathrm{m}$. This was much greater than $100 \mu \mathrm{m}$, the limiting size below which survival could not be facilitated [Khosravi Zanjani et al., 2014]. Nevertheless, we found that the applied encapsulation did not improve the overall survival of the studied probiotics using neither of the probiotic strains nor the encapsulation approaches. These data do not generally agree well with the literature reviewed by Shori [2017]. Also, in recently published research papers [Yeung et al., 2016; Zaeim et al., 2017], alginate-chitosan encapsulation is claimed an effective means of protecting probiotics. Nevertheless, overall results show that this protection is limited. For example, Yeung et al. [2016] encapsulated $B$. infantis UMA299 into alginatechitosan using injection-gelation method and observed that the encapsulation provided improved protection to stomach juice (by $1.3 \log$ cfu compared to control), but not duodenum juice. In the present study, we also noted a significant improvement of protection of L. casei W56 in stomach juice compared to control when probiotic powder was encapsulated (by $3.2 \log \mathrm{cfu}$; calculated based on data from Figure 2 when corrected for the dilution factor resulting from the addition of the digestive juice to the sample). Nevertheless overall survival of $L$. casei W56 did not improve due to increased sensitivity of the encapsulated probiotic to duodenal and ileal juice (increase of log reduction compared to control by 1.5 and $3.2 \log$ cfu, respectively; calculated based on data from Figure 2 when corrected for the dilution factor resulting from the addition of the digestive juice to the sample). Furthermore, for encapsulates containing broth-grown
L. casei W56, survival in the stomach or any other digestive stage was not improved, and total log reduction was significantly greater compared to the control (Figure 3 ).

In another study, authors investigated the survival of L. plantarum ATCC 8014 encapsulated into alginate-chitosan using electrospraying [Zaeim et al., 2017]. They have found that overall the survival of probiotic was improved compared to free cells, but only by $\sim 0.9 \log$ cfu. This improvement was of a similar magnitude to the one observed for broth-grown, encapsulated B. lactis W52 (total log reduction decreased compared to control by $1.1 \log \mathrm{cfu}$ ).

\section{The effect of the presence and concentration of prebiotics on the survival of probiotics during in vitro digestion}

It has been shown that probiotic survival in the digestive juices may be improved using prebiotics proportionally to the applied prebiotic concentration [Haghshenas et al., 2015; Kingwatee et al., 2014; Sanchez et al., 2014]. In the present study, we did not observe the improvement of survival in digestive liquids with increased concentration of either inulin or FOS. Furthermore, the total log reduction seen upon application of prebiotics in our in vitro digestion, decreased by the maximum of $1.4 \log$ cfu compared to the control (for B. lactis W52 with $0.1 \%$ FOS). Clearly, this decrease was lower compared to what could be expected based on the literature (upon application of prebiotic concentration $\geq 0.1 \%$ the $\log$ reduction decreased from 2 to $\sim 4 \log$ cfu in studies by Haghshenas et al. [2015], Kingwatee et al. [2014], and Sanchez et al. [2014]).

It is not clear why in this study we have not seen substantial improvement of probiotic survival in the presence of prebiotics. One of the possible reasons could be the ability of probiotics to metabolise substances as an energy source. In this study, we did not focus on probiotic metabolism but on the evaluation of different approaches for the improvement of probiotic survival. Nevertheless, obtained results and published literature data highlight that probiotic metabolism could be one of the factors contributing to probiotic stress resistance and should be a subject of further research [Wang et al., 1999; Corcoran et al., 2005].

\section{CONCLUSIONS}

In the present study we have reported survivability of L. casei W56 and B. lactis W52 in the presence and absence of food matrices, prebiotics and upon encapsulation during simulated passage through selected parts of the human digestive tract. Although improvements in the digestive survival of $B$. lactis W52 were achieved by application of milk and fermented milk, no solution seemed to improve viability of L. casei W56. Hence, neither of the examined methods could be recommended as a universal solution for the improvement of probiotic survival during passage through upper parts of the digestive tract.

Findings presented in this work suggested that in a choice of suitable method for the digestive survival improvement, probiotic characteristics play an important role. In the course of this study we have found that the studied probiotics featured a different survival behaviour. While L. casei W56 was 
sensitive to stomach juice, $B$. lactis W52 was relatively resistant to stomach juice but declined in the presence of duodenum juice. Interestingly, B. lactis W52 survived the digestion well in the presence of matrices that contained fat (milk and fermented milk, 3.6\%). This finding indicated that the interaction of bile with fat might have minimised the losses of B. lactis W52 in the duodenum juice.

Another important characteristic of probiotics that may improve their ability to survive through upper digestive tract is the utilisation of the matrix components as an energy source. Prebiotics, starch as well as metabolizable sugars may be used by probiotics as food and consequently provide energy for the removal of protons from cells (as shown for glucose by Corcoran et al. [2005]), improving resistance to gastric acid. Although the study presented here did not focus on the characterisation of probiotic metabolism, obtained results highlighted that the ability to utilise surrounding matrix as a food source might be of key interest if the improvement of the probiotic survivability in the human digestive tract is sought.

Our results suggested that probiotic manufacturers could consider focusing on the development of suspension protocols for probiotic powders. Currently, the general guidance for a suspension of probiotic powder is to mix it with water prior to ingestion. Based on the results presented here, we could recommend using whole milk instead. Further research into optimisation of such protocols looking at different, acid-sensitive probiotics may be of benefit.

This research highlighted knowledge gaps in understanding mechanisms governing probiotic survival in the upper gastrointestinal tract. Optimisation of probiotic survival in studies investigating health benefits of probiotics could address to date observed discrepancies between the reports (as noted by e.g. Kasińska \& Drzewoski [2015]).

\section{RESEARCH FUNDING}

We would like to acknowledge the National Centre for Food Manufacturing and the Undergraduate Research Opportunities Scheme funding awarded by the University of Lincoln for financial support for this project.

\section{ACKNOWLEDGEMENTS}

We thank Winclove Probiotics and especially Dr Saskia van Hemert for providing us study materials and professional advice on this work. We are also appreciative of help received from Ruth Britton and Sophie Bowers during training and work on the laboratory trials. Finally, we would like to thank the Jan Dlugosz University in Czestochowa for covering article publication charges.

\section{CONFLICT OF INTEREST}

Authors declare no conflict of interest.

\section{REFERENCES}

1. Begley, M., Gahan, C.G.M., Hill, C. (2005). The interaction between bacteria and bile. FEMS Microbiology Reviews, 29(4), 625-651.

2. Bezkorovainy, A. (2001). Probiotics: determinants of survival and growth in the gut. The American Journal of Clinical Nutrition, 73(2), 399s-405s.

3. Burgain, J., Gaiani, M., Linder, M., Scher, J. (2011). Encapsulation of probiotic living cells: From laboratory scale to industrial applications. Journal of Food Engineering, 104(4), 467-483.

4. Coghetto, C.C., Brinques, G.B., Siqueira, N.M., Pletsch, J., Soares, R.M.D., Ayub, M.A.Z. (2016). Electrospraying microencapsulation of Lactobacillus plantarum enhances cell viability under refrigeration storage and simulated gastric and intestinal fluids. Journal of Functional Foods, 24, 316-326.

5. Corcoran, B.M., Stanton, C., Fitzgerald, G.F., Ross, R.P. (2005). Survival of probiotic Lactobacilli in acidic environments is enhanced in the presence of metabolizable sugars. Applied and Environmental Microbiology, 71(6), 3060-3067.

6. Fávaro-Trindade, C.S., Grosso, C.R.F. (2002). Microencapsulation of L. acidophilus (La-05) and B. lactis (Bb-12) and evaluation of their survival at the $\mathrm{pH}$ values of the stomach and in bile. Journal of Microencapsulation, 19(4), 485-494.

7. Fredua-Agyeman, M., Gaisford, S. (2015). Comparative survival of commercial probiotic formulations: tests in biorelevant gastric fluids and real-time measurements using microcalorimetry. Beneficial Microbes, 6(1), 141-151.

8. Govender, M., Choonara, Y.E., Kumar, P., Toit, L.C. du, Vuuren, S., Pillay, V. (2014). A review of the advancements in probiotic delivery: conventional vs. non-conventional formulations for intestinal flora supplementation. AAPS PharmSciTech, 15(1), 29-43.

9. Haghshenas, B., Nami, Y., Haghshenas, M., Barzegari, A., Sharifi, S., Radiah, D., Rosli, R., Abdullah, N. (2015). Effect of addition of inulin and fenugreek on the survival of microencapsulated Enterococcus durans 39C in alginate-psyllium polymeric blends in simulated digestive system and yogurt. Asian Journal of Pharmaceutical Sciences, 10(4), 350-361.

10. ISO 15214:1998 Microbiology of food and animal feeding stuffs - Horizontal method for the enumeration of mesophilic lactic acid bacteria - Colony-count technique at 30 degrees C.

11. Kasińska, M.A., Drzewoski, J. (2015). Effectiveness of probiotics in type 2 diabetes: a meta-analysis. Polskie Archiwum Medycyny Wewnętrznej, 125(11), 803-813.

12. Kellow, N.J., Coughlan, M.T., Reid, C.M. (2014). Metabolic benefits of dietary prebiotics in human subjects: a systematic review of randomised controlled trials. British Journal of Nutrition, $111(7), 1147-1161$.

13. Khosravi Zanjani, M.A., Ghiassi Tarzi, B., Sharifan, A., Mohammadi, N. (2014). Microencapsulation of probiotics by calcium alginate-gelatinized starch with chitosan coating and evaluation of survival in simulated human gastro-intestinal condition. Iranian Journal of Pharmaceutical Research, 13(3), 843-852.

14. Kingwatee, N., Apichartsrangkoon, A., Chaikham, P. (2014). Survivability and metabolic activity of Lactobacillus casei 01 in- 
corporating lychee juice plus inulin under simulated gastrointestinal environment. International Food Research Journal, 21 (1), 83-89.

15. Lo Curto, A., Pitino, I., Mandalari, G., Dainty, J.R., Faulks, R.M., John Wickham, M.S. (2011). Survival of probiotic lactobacilli in the upper gastrointestinal tract using an in vitro gastric model of digestion. Food Microbiology, 28(7), 1359-1366.

16. Marteau, P., Minekus, M., Havenaar, R., Huis in't Veld, J.H. (1997). Survival of lactic acid bacteria in a dynamic model of the stomach and small intestine: validation and the effects of bile. Journal of Dairy Science, 80(6), 1031-1037.

17. Park, H.K., So, J.S., Heo, T.R. (1995). Acid adaptation promotes survival of Bifidobacterium breve against environmental stresses. Foods and Biotechnology, 4(4), 226-230.

18. Sanchez, M., Darimont, C., Drapeau, V., Emady-Azar, S., Lepage, M., Rezzonico, E., Ngom-Bru, C., Berger, B., Philippe, L., Ammon-Zuffrey, C., Leone, P., Chevrier, G., St-Amand, E., Marette, A., Doré, J., Tremblay, A. (2014). Effect of Lactobacillus rhamnosus CGMCC1.3724 supplementation on weight loss and maintenance in obese men and women. The British Journal of Nutrition, 111 (8), 1507-1519.

19. Segale, L., Giovannelli, L., Mannina, P., Pattarino, F. (2016). Calcium alginate and calcium alginate-chitosan beads containing celecoxib solubilized in a self-emulsifying phase. Scientifica, 2016, art. no. 5062706.

20. Shori, A.B. (2017). Microencapsulation improved probiotics survival during gastric transit. HAYATI Journal of Biosciences, 24(1), 1-5.

21. Sohail, A., Turner, M.S., Coombes, A., Bostrom, T., Bhandari, B. (2011). Survivability of probiotics encapsulated in alginate gel microbeads using a novel impinging aerosols method. International Journal of Food Microbiology, 145(1), 162-168.
22. Spano, G., Massa, S. (2006). Environmental stress response in wine lactic acid bacteria: beyond Bacillus subtilis. Critical Reviews in Microbiology, 32(2), 77-86.

23. Sumeri, I., Arike, L., Stekolstsikova, J., Uusna, R., Adamberg, S., Adamberg, K., Paalme, T. (2010). Effect of stress pretreatment on survival of probiotic bacteria in gastrointestinal tract simulator. Applied Microbiology and Biotechnology, 86(6), 1925-1931.

24. Timmerman, H.M., Niers, L.E.M., Ridwan, B.U., Koning, C.J.M., Mulder, L., Akkermans, L.M.A., Rombouts, F.M., Rijkers, G.T. (2007). Design of a multispecies probiotic mixture to prevent infectious complications in critically ill patients. Clinical Nutrition, 26(4), 450-459.

25. Tompkins, T.A., Mainville, I., Arcand, Y. (2011). The impact of meals on a probiotic during transit through a model of the human upper gastrointestinal tract. Beneficial Microbes, 2(4), 295-303.

26. Wang, X., Brown, I.L., Evans, A.J., Conway, P.L. (1999). The protective effects of high amylose maize (amylomaize) starch granules on the survival of Bifidobacterium spp. in the mouse intestinal tract. Journal of Applied Microbiology, 87(5), 631-639.

27. Yeung, T.W., Üçok, E.F., Tiani, K.A., McClements, D.J., Sela, D.A. (2016). Microencapsulation in alginate and chitosan microgels to enhance viability of Bifidobacterium longum for oral delivery. Frontiers in Microbiology, 7, art. no. 494.

28. Zaeim, D., Sarabi-Jamab, M., Ghorani, B., Kadkhodaee, R., Tromp, R.H. (2017). Electrospray assisted fabrication of hydrogel microcapsules by single- and double-stage procedures for encapsulation of probiotics. Food and Bioproducts Processing, 102 (Supplement C), 250-259.

Submitted: 6 January 2020. Revised: 25 March and 3 April 2020. Accepted: 7 April 2020. Published on-line: 19 June 2020. 
